\title{
Review Article \\ Systematic Review on Chinese Herbal Medicine Induced Liver Injury
}

\author{
Peng Zhang, ${ }^{1}$ Yongan Ye, ${ }^{2}$ Xianzhao Yang, ${ }^{3}$ and Yuntao Jiao ${ }^{1}$ \\ ${ }^{1}$ The First Clinical Medical School, Beijing University of Chinese Medicine, Beijing, China \\ ${ }^{2}$ Department of Gastroenterology and Hepatology, Beijing University of Chinese Medicine Affiliated Dongzhimen Hospital, Beijing, \\ China \\ ${ }^{3}$ Department of Infectious Disease, Beijing University of Chinese Medicine Affiliated Dongzhimen Hospital, Beijing, China
}

Correspondence should be addressed to Yongan Ye; yonganye@yahoo.com

Received 10 June 2016; Revised 22 July 2016; Accepted 1 August 2016

Academic Editor: Victor Kuete

Copyright (C) 2016 Peng Zhang et al. This is an open access article distributed under the Creative Commons Attribution License, which permits unrestricted use, distribution, and reproduction in any medium, provided the original work is properly cited.

Background. In recent years, with the popularity of $\mathrm{CHM}$, its hepatotoxicity has also been increasingly noticed. However, there are still veils on causative herbs and clinical characteristics. Aim. To systematically review data on CHM induced liver injury with particular focus on causative herbs and clinical characteristics. Methods. Using terms related to CHM and liver injury, PubMed and three Chinese electronic databases were searched, which was limited to the past 5 years. Publications meeting our eligibility criteria were included and further analyzed. Results. In total, 4 single herbs, 21 patent drugs, and 4 decoctions were reported to be of hepatotoxicity, with He-Shou-Wu being the most common one (65/114). Dang-Gui and other 5 herbs were the most common ingredients of patent drugs and decoctions. All patients were assessed using the RUCAM scale, with 26 being highly probable and 28 being probable. For these 54 cases, the latent period was 30 (47) days, and 81.48\% were labeled as hepatocellular injuries. Most patients (96.3\%) recovered, apart from the fact that one died and one is receiving liver transplantation. Conclusions. CHM should be used carefully for hepatotoxicity. Liver injury from CHM is similar to that from conventional medicines in clinical characteristics. Details about causative herbs should be illustrated, and more RUCAM should be used in future.

\section{Introduction}

Traditional Chinese medicine (TCM), originated in ancient China, has been widely used to treat diseases for thousands of years, using Chinese herbal medicine (CHM), acupuncture, moxibustion, and other body practices. In recent decades, TCM has been increasingly popular around the world [14]. As the main part of TCM, CHM is usually combined in formulas and taken orally as decoction, powders, and other forms, following TCM theories. Although CHM plays an important role in health care, more and more liver injury cases from CHM are reported. While the exact number is unavailable, nearly $20 \%$ of drug-induced liver injuries (DILI) were due to CHM in China $[5,6]$. Therefore, it is of great importance to study CHM induced liver injury.

To learn CHM induced liver injury, some questions are inevitable, namely, which herbs can lead to liver injury specifically, when it will cause liver injury, and what the clinical characteristics are. Though some reviews [7-10] have been published to give detailed information, they did not take Chinese electronic database into account, in which large amounts of data about use and adverse events of CHM are found. While these reviews did put an emphasis on causality assessment by the Roussel Uclaf Causality Assessment Method (RUCAM) scale [11] and positive reexposure tests, they paid little attention to strict definition of hepatotoxicity. A low threshold of liver enzyme values may allow cases with nonspecific increases in.

To help clinicians and TCM practitioners know more about and avoid CHM induced liver injury, we systematically reviewed publications, giving a list of Chinese herbal medicines with possible hepatotoxicity and summarizing associated clinical characteristics. Since more attention has been paid to DILI in recent years and the diagnosis has also developed both in China and the world, our review focused on literature of the past 5 years. 


\section{Methods}

2.1. Literature Search. Our review was planned and performed in conformity with Cochrane Handbook for Systematic Reviews of Interventions [12] and Preferred Reporting Items for Systematic Review and Meta-Analysis (PRISMA) statement [13], which was also published on the PROSPERO register, with a registration number being CRD42016036053. A literature search in PubMed database and three Chinese electronic databases, including China National Knowledge Infrastructure (CNKI), Wan Fang database, and VIP database, was independently carried out by two investigators, using the terms "herb*," "Chinese medicine," "traditional medicine," and "complementary and alternative medicine" and "liver injury," "hepatotoxicity," "liver disease," and "hepatitis." The maximal number of articles was obtained using terms in all possible combinations. The search was limited to English and Chinese language articles and restricted between 2011 and March 1, 2016.

2.2. Eligibility Criteria. Articles included have to meet the following criteria. (1) Studies on human subjects are included. (2) Liver injury is specifically induced by Chinese herbal medicines, which include single herbs, patent drugs, and decoctions made up of herbal ingredients. Herbs included should be usually used by TCM practitioners or officially listed in the Chinese Pharmacopoeia [14]. (3) Liver injury is defined as elevations of ALT above 5 times the upper limit of normal (ULN) and/or ALP above 2 times ULN. If ALT > $5 \mathrm{ULN}$ and ALP $\leq \mathrm{ULN}$ or if both ALT and ALP are elevated, $R \geq 5$, the liver injury is hepatocellular. If ALP $>2 \mathrm{ULN}$ and ALT $\leq$ ULN or if both ALT and ALP are elevated, $R \leq 2$, the liver injury is cholestatic. If ALT $>5 N$ and ALP $>N$ and $2<R<5$, the liver injury is mixed. (4) Causality assessment is done using the RUCAM scale [11], with a score no less than 3 points. If the pattern of liver injury is hepatocellular, a subtype of RUCAM for hepatocellular injury is used, and if it is cholestatic or mixed, a subtype for the cholestatic or mixed injury is performed.

2.3. Study Selection and Data Extraction. Included articles were independently reviewed by two authors, based on title/abstract firstly and full-text secondly. During the process of full-text selection, disagreements were resolved by discussion, and if an agreement could not be reached, a third author would make a decision. The following data were recorded: causative herbs, demographic information, regional distribution, primary diseases, usage and dosage, latent period, laboratory results, pattern of liver injury, causality assessment, reexposure results, and clinical outcomes.

2.4. Statistical Analysis. A descriptive analysis was used. Enumeration data was described with frequency distribution, while measurement data was described with centralized tendency. Normally distributed data was described as Mean \pm Standard Deviation, while data obeying abnormal distribution was presented as Median (Interquartile Range). All statistical analyses were performed using SPSS software (version 20.0). Cases with incomplete clinical information were also included in this review, but only those with welldefined values for each parameter were included in the statistical analysis.

\section{Results}

3.1. Literature Selection and Characteristics. The initial search produced 4363 articles (Figure 1), of which 865 were excluded for duplicates and 3299 were eliminated as animal studies, experiments in vitro, reviews, and studies irrelevant to CHM. After further evaluation, 17 articles [15-31] fulfilled the eligibility criteria, of which 14 were case reports, 2 were case series, and 1 was cross-sectional study. 114 cases were included in total, of which 83 cases were from China, 26 were from Korea, 4 were from the United States, and 1 was from Japan. Detailed information of CHM, like locality and specific taxa, was not mentioned in all 17 articles, and only 4 of 17 described correct scientific names of herbs [18, 19, 21, 22]. Among six articles reporting liver injury attributed to Chinese patent drugs [15-17, 21, 23, 29], there was one [29] without detailed herbal ingredients, six without herbal contents, three [15, $23,29]$ without recommended dosage and usage, and three $[16,23,29]$ without brand names or manufacturers. Only 1 of 4 articles reporting decoctions mentioned detailed herbal dosage [30].

3.2. Identification of CHM with Reported Hepatotoxicity. In total, 4 kinds of single herbs, 21 patent drugs, and 4 decoctions made up of multiple herbs were reported to have caused liver injury, including He-Shou-Wu [Reynoutria multiflora (Thunb.) Moldenke], Cang-Er-Zi [Xanthium strumarium subsp. sibiricum (Patrin ex Widder) Greuter], Huang-Yao$\mathrm{Zi}$ (Dioscorea bulbifera L.), Lei-Gong-Teng (Tripterygium wilfordii Hook. f.), Yang Xue Sheng Fa Jiao Nang, Bai Dian Feng Jiao Nang, Xiao Yin Pian, Qu Bai Ba Bu Pian, Bu Shen Sheng Fa Tang, Ze Qi Chong Ji, Xian Ling Gu Bao Jiao Nang, Gu Kang Jiao Nang, Zhuang Gu Jiao Nang, Ling Zhi Yi Shou Jiao Nang, Ling Zhi Jiao Nang, Hui Chun Ru Yi Jiao Nang, Ru Bi San, ShuXiong Jiao Nang, Zeng Sheng Ping, Long Bi Shu, Zhi Xue Jiao Nang, Move Free, Ban Tu Wan, Kamishoyosan, Qi Bao Mei Ran Wan, herbal extracts containing Hu-JiSheng [Viscum coloratum (Kom.) Nakai] and Ye-Ge (Pueraria montana var. lobata (Willd.) Sanjappa \& Pradeep), herbal tea containing Kelp, and two decoctions consisting of CHM. Detailed information is listed in Table 1. Of the total 114 cases, liver injury caused by $\mathrm{He}-\mathrm{Shou}-\mathrm{Wu}$ accounted for 65 , which was the most common one in our review. With regard to primary diseases of included cases, dermatosis, grey hair, and alopecia took up 11/30, which was the largest proportion. Other applications involved were osteoarthrosis $(5 / 30)$, health promotion $(3 / 30)$, diabetes mellitus $(2 / 30)$, and mammary gland disorders (2/30). In addition, a further analysis about ingredients of patent drugs and decoctions was performed. Totally, 72 kinds of herbs were involved in 4 decoctions and 8 patent drugs with detailed ingredients, reported in articles or the Chinese Pharmacopoeia, of which Dang-Gui [Angelica sinensis (Oliv.) Diels], He-Shou-Wu, 


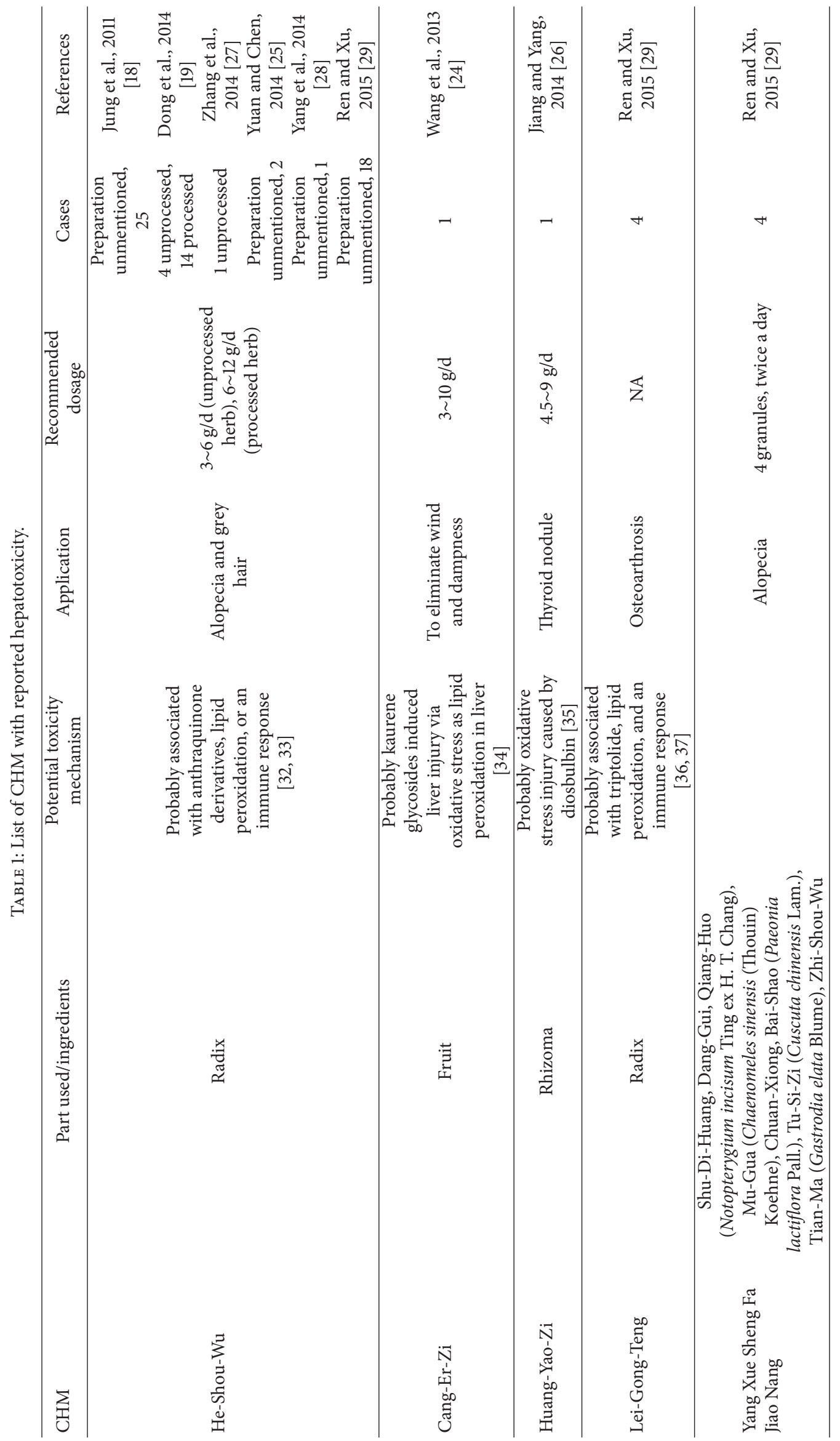




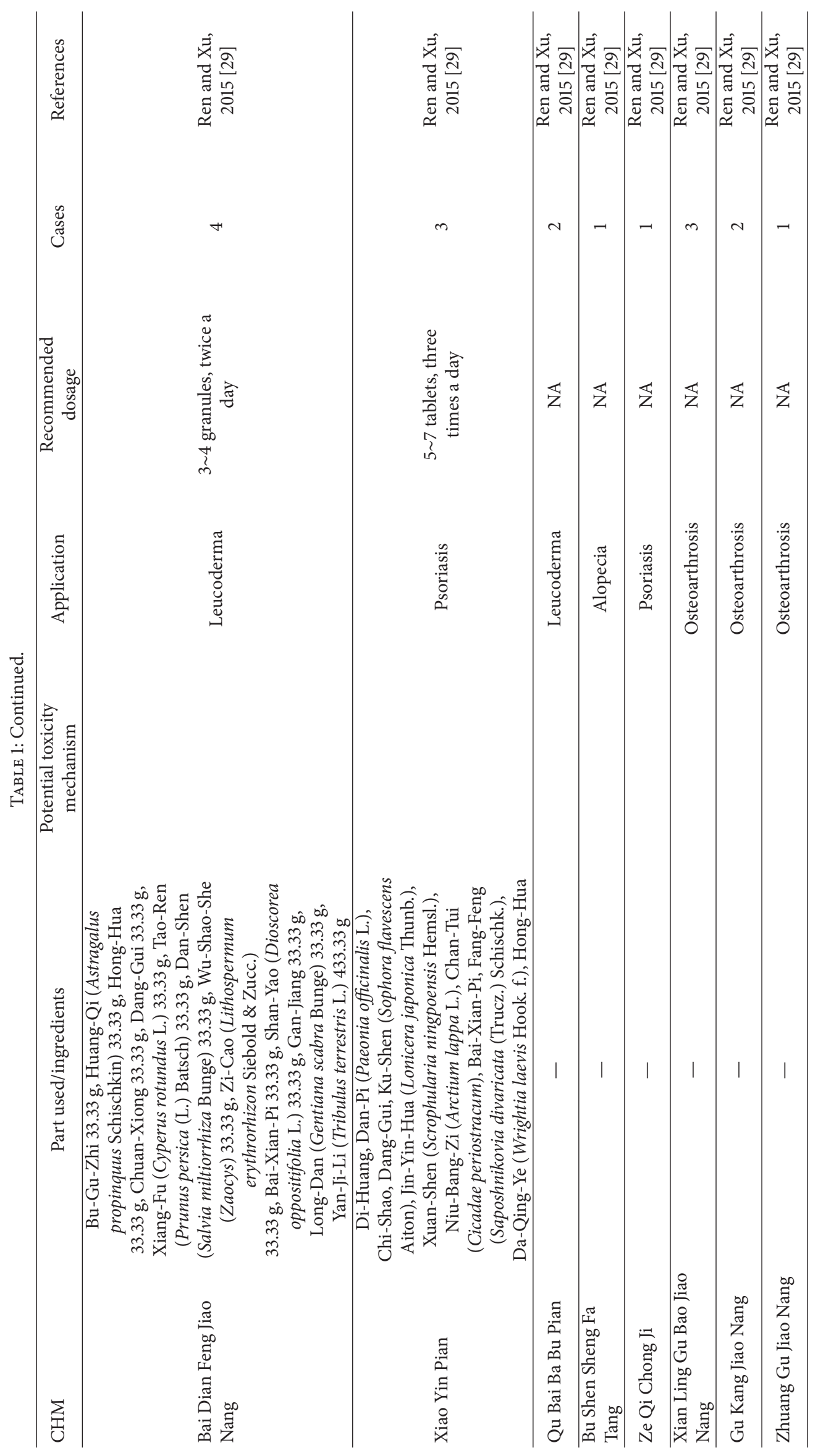




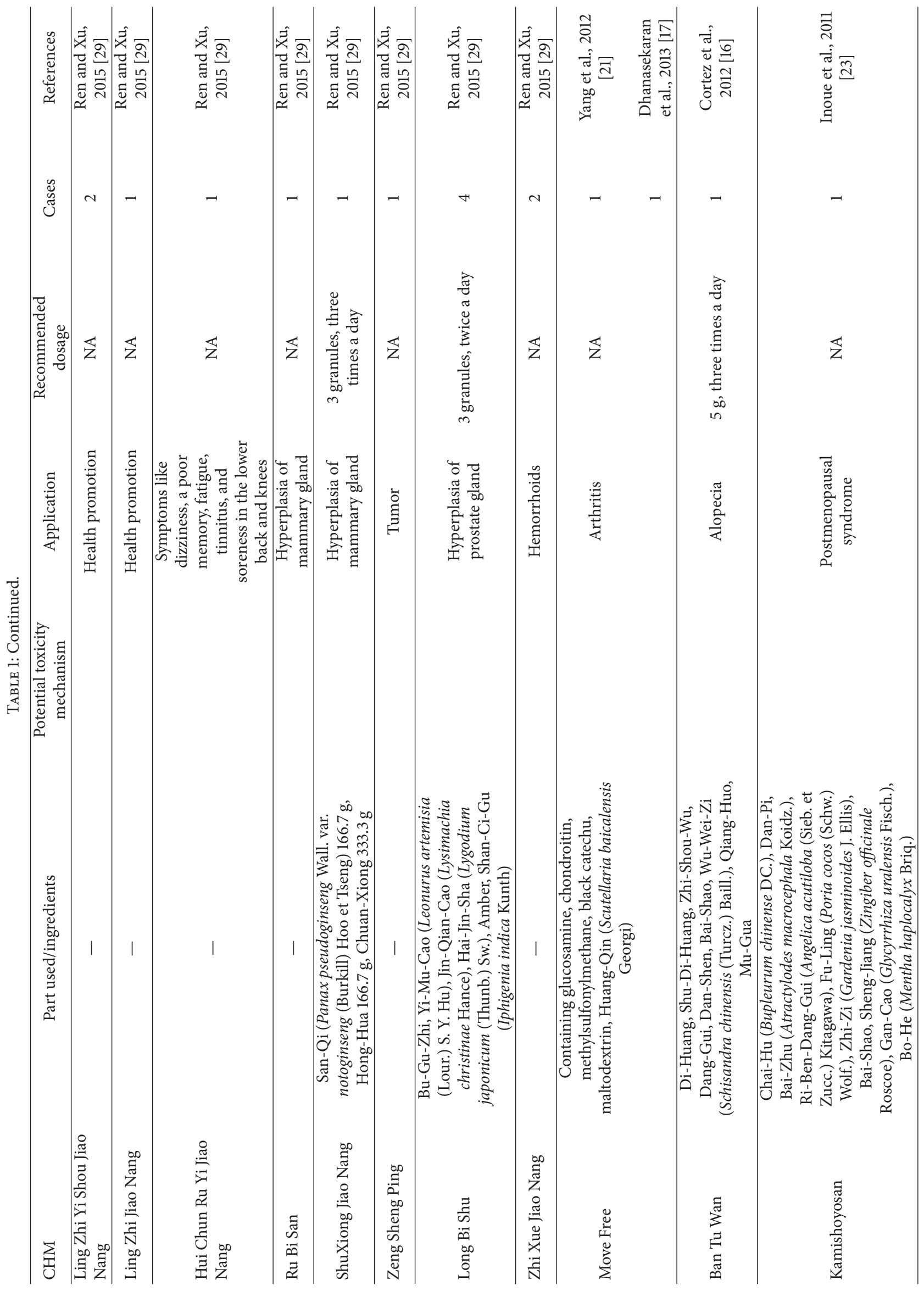




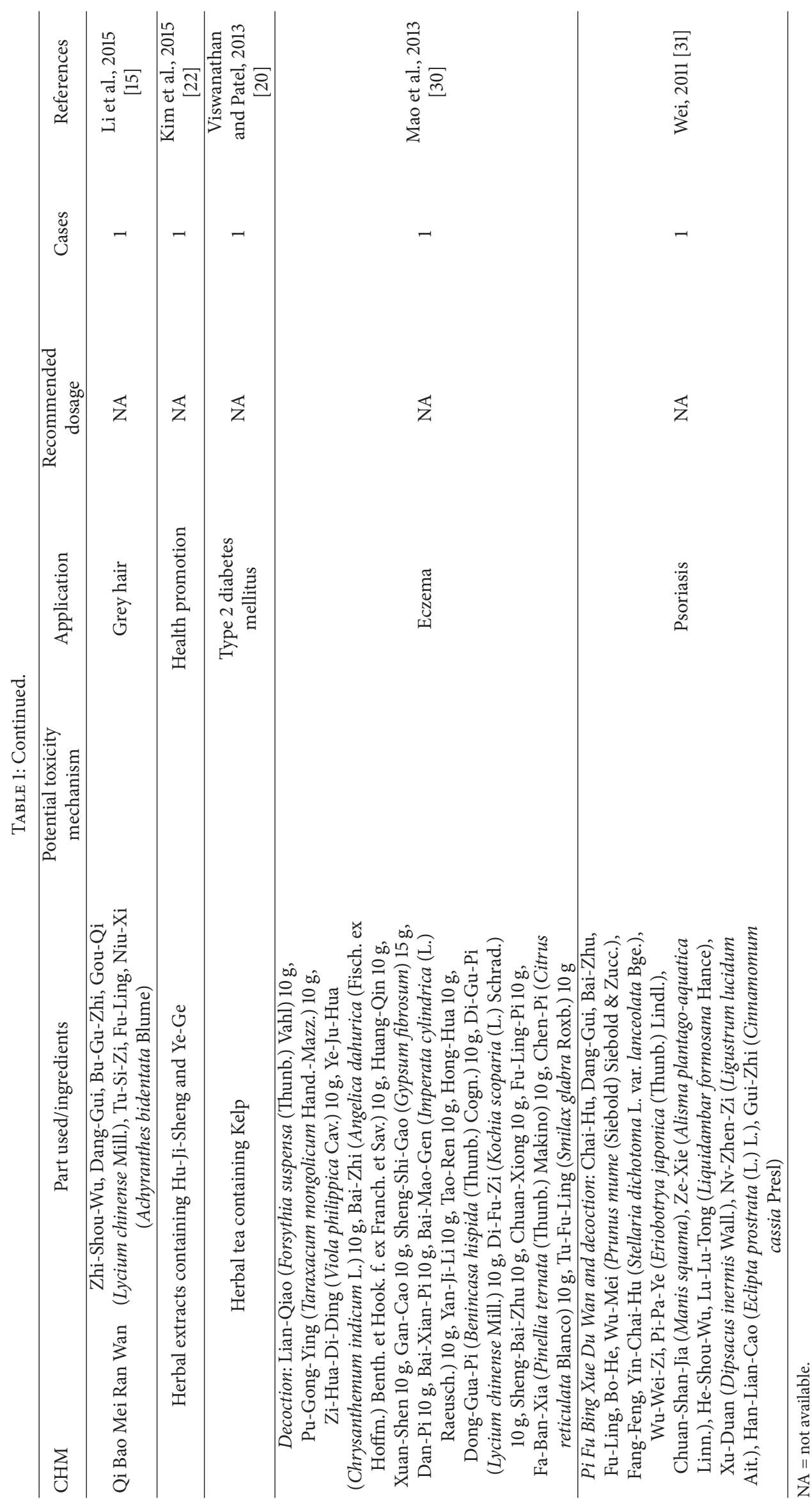




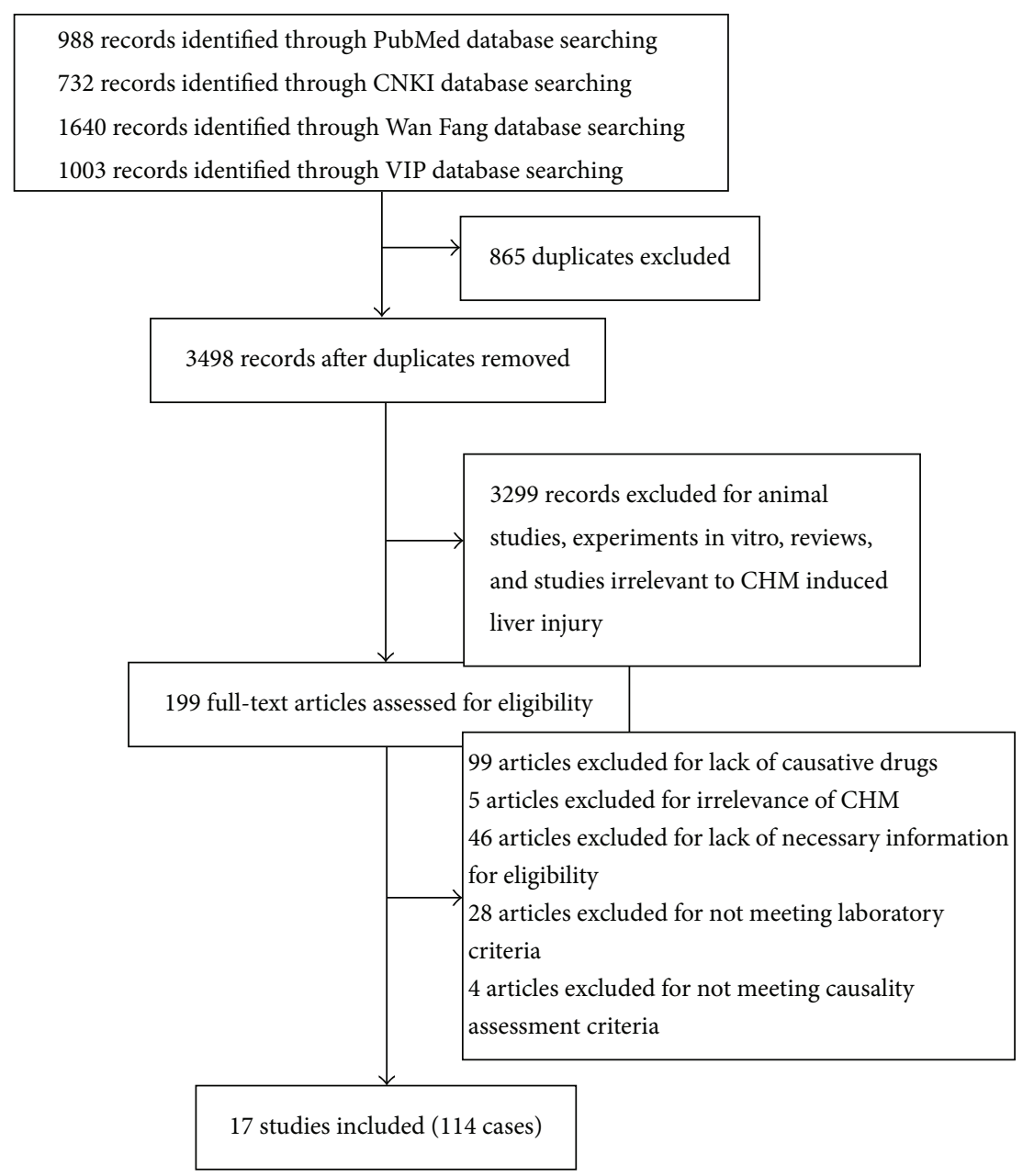

FIgURE 1: Flow chart of literature selection.

Di-Huang [Rehmannia glutinosa (Gaertn.) DC.], ChuanXiong (Ligusticum striatum DC.), Hong-Hua (Chelonopsis pseudobracteata var. rubra C. Y. Wu \& H. W. Li), Bai-Xian$\mathrm{Pi}$ (Dictamnus albus L.), and Bu-Gu-Zhi [Psoralea cordata (Thunb.) Salter] were the most common ones. Details are shown in Figure 2.

3.3. Causality Assessment. Causality assessment is necessary for the diagnosis of CHM induced liver injury. While expert consensus opinion and the RUCAM scale are considered as preferred algorithms to establish causality in suspected herbinduced liver injury (HILI), the former one is not widely available since it is cumbersome, costly, and time consuming. RUCAM scale is much more widely used by clinicians and researchers, which is structured, quantitative, and validated for liver injury. Of the total 17 articles included, 7 directly used the RUCAM scale to assess causality, while the other 10 articles provided associated information, based on which RUCAM can be performed. Four articles [9, 25, 28, 31] explicitly excluded HEV by serology tests, and four [20, 22, $26,30]$ demonstrated exclusion of viral hepatitis without specific mention of HEV. Fifty-six cases of one article [29], scored no less than 3 points, were not supplied with detailed information and cannot be graded by RUCAM. Other cases were grouped into different likelihood levels, with 26 being highly probable (score $>8$ ), 28 being probable (6-8), and 4 being possible (3-5). In a further analysis, 54 cases with more than 6 points of RUCAM were identified, in which He-ShouWu, Huang Yao Zi, Move Free, Kamishoyosan, Qi Bao Mei Ran Wan, herbal extracts containing Hu-Ji-Sheng and YeGe, herbal tea containing Kelp, and a decoction consisting of CHM were causative. Since these herbs were more likely to cause liver injury, more attention should be paid. Furthermore, one case attributed to $\mathrm{He}-\mathrm{Shou}-\mathrm{Wu}$ was reported with a positive reexposure result, which accorded with the criteria of reexposure [11], while another case caused by kamishoyosan did not supply detailed ALT level of the first time, which, consequently, could not be diagnosed as reexposure.

3.4. Clinical Characteristics. Patients' features and clinical characteristics of liver injury from CHM were summarized. Of the 58 cases with detailed information, all 54 with probable or highly probable causality grading were included. Thus, we focused on these 54 cases and showed their clinical characteristics. While 35 were male and 19 were female, there was an average age of $47.13 \pm 12.24$ years, ranging 


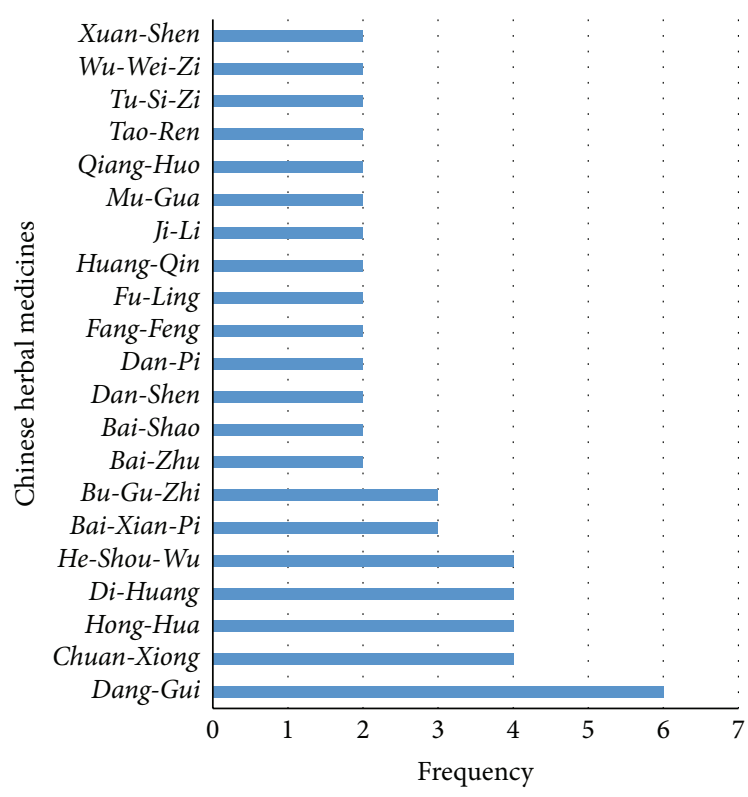

FIGURE 2: Frequency distribution of herbal ingredients of patent drugs and decoctions was performed, and herbs used more than once were given in the figure.

from 17 to 78. The latent period was 30 (47) days, which is consistent with DILI [38]. Nine of eleven cases with welldefined information of actual and recommended dosage were involved in excessive intake of $\mathrm{CHM}$, indicating that excessive use may be related to the incidence of liver injury. Concerning laboratory parameters, the average serum levels of ALT and AST were $1246(824.25) \mathrm{IU} / \mathrm{L}$ and $931.52 \pm 598.36 \mathrm{IU} / \mathrm{L}$, respectively, and the average serum level of ALP was 225.08 \pm $170.66 \mathrm{IU} / \mathrm{L}$. The high laboratory values may be due to our restriction criteria for hepatotoxicity, which helps eliminate unspecific liver enzymes' increases and substantiate causality at higher probability. Cases were further identified by the pattern of liver injury, consisting of forty-four $(81.48 \%)$ of hepatocellular injuries, eight (14.81\%) of mixed injuries, and two (3.7\%) of cholestatic injuries. Most of patients recovered from CHM induced liver injury, with a percentage of $96.3 \%$, apart from the fact that one died and one is receiving liver transplantation. Additional details are available in Table 2.

\section{Discussion}

DILI is one of the most common causes of hepatitis in the world. According to some studies, DILI accounted for about $11 \%$ of acute liver failure cases [39, 40]. As a result of popularity, liver injury induced by CHM is on the increase around the world. To help TCM practitioners avoid CHM induced liver injury and supply clinicians with more associated data, we systematically reviewed publications, focusing on developments in the recent 5 years.

Characteristics of included cases were summarized, while problems in the original papers were brought up. Detailed information about CHM, like locality, botanical classification, brand names, detailed contents, and usage and dosage, was unavailable in most publications, which is of great importance to improve the reliability of studies. CHM with reported hepatotoxicity were identified, of which He-Shou$\mathrm{Wu}$ attracted our attention. He-Shou-Wu is one of the most popular CHM, officially listed in the Chinese Pharmacopoeia. According to Ben Cao Gang Mu (Compendium of Materia Medica), an ancient book recording therapeutic effects of $\mathrm{CHM}, \mathrm{He}-\mathrm{Shou}-\mathrm{Wu}$ is usually used to treat alopecia and white hair by nourishing the liver and kidneys. In recent years, with the increasing use of it, numbers of hepatotoxicity cases have been reported [18, 19, 41, 42]. Although efforts have been made, the toxicity mechanism is still not fully elucidated. Probably, the hepatotoxicity is related to some bioactive compounds, anthraquinone derivatives [32, 43]. Based on TCM theory, processing is believed to be able to reduce the toxicity of herbs, including He-Shou-Wu, but both processed and unprocessed He-Shou-Wu were reported to cause liver injury in publications. The phenomenon does not necessarily mean that preparation is of no use to hepatotoxicity. Since the use of the processed one is much more common than the unprocessed one and the hepatotoxicity can be affected by many other factors, the relationship between processing and hepatotoxicity cannot be concluded for now. Our review also indicated that hepatotoxicity was much more common with dermatosis and osteoarthrosis, which reminds dermatologists or orthopedists of avoiding those recorded herbs. Since people increasingly focus on health care, CHM used for health promotion also should be taken carefully.

It is worth noting that formulae consisting of multiple herbs, including patent drugs and decoctions, prevail in $\mathrm{CHM}$ with potential hepatotoxicity. In the view of TCM, liver injury from these drugs and decoctions is often related to some ingredients with hepatotoxicity. After a review of related literature, in our results, $\mathrm{He}-\mathrm{Shou}-\mathrm{Wu}, \mathrm{Bai}-\mathrm{Xian}-\mathrm{Pi}$, and $\mathrm{Bu}-$ $\mathrm{Gu}$-Zhi have been reported to be of hepatotoxicity [10, 44], while Dang-Gui, Di-Huang, Chuan-Xiong, and Hong-Hua have not been, among which Dang-Gui, Di-Huang, and Hong-Hua have been reported to be with hepatoprotective effect [45-47]. These three herbs are often used in TCM prescriptions, especially used for skin and gynecological diseases, which may account for their high frequency. TCM practitioners always use a prescription to treat diseases, which blends together a number of herbs with specific functions. Sometimes, prescription compatibility may also play an important role in attenuating adverse events of CHM [48]. It is definitely necessary for clinicians to supply detailed ingredients and contents in articles as far as possible. However, during literature selection, lots of articles were excluded for lack of detailed information of multiple ingredients, which is the same as situation on the online websites, LiverTox [49] and HepaTox [50].

Since syndrome differentiation and individualized treatment are main features of TCM, excessive use of CHM is necessary and universal sometimes. It is also essential to further analyze its relationship with hepatotoxicity on the basis of more data. In our results, the values of liver tests were relatively high, which may be related to our strict inclusion criteria. The majority of cases were grouped into 


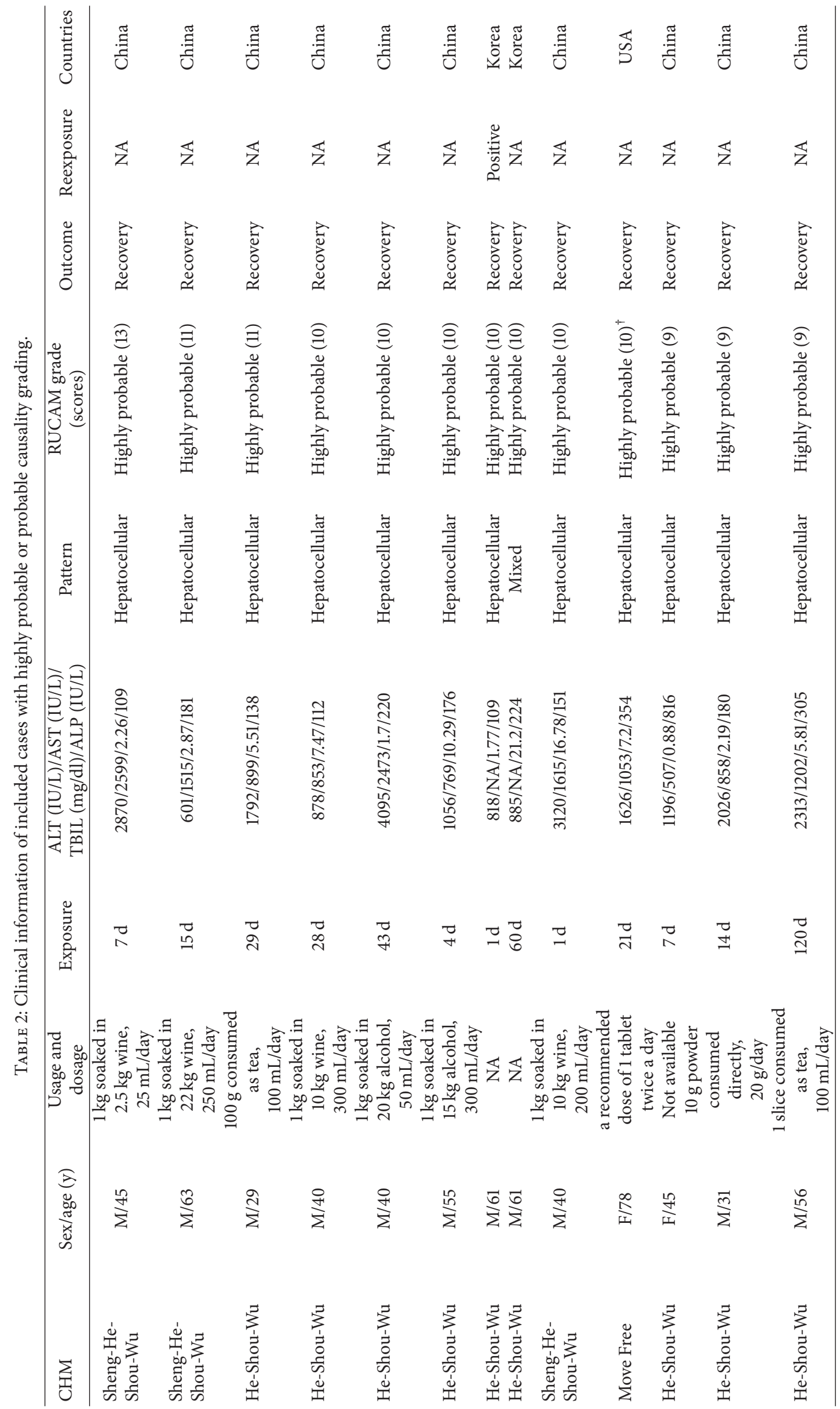




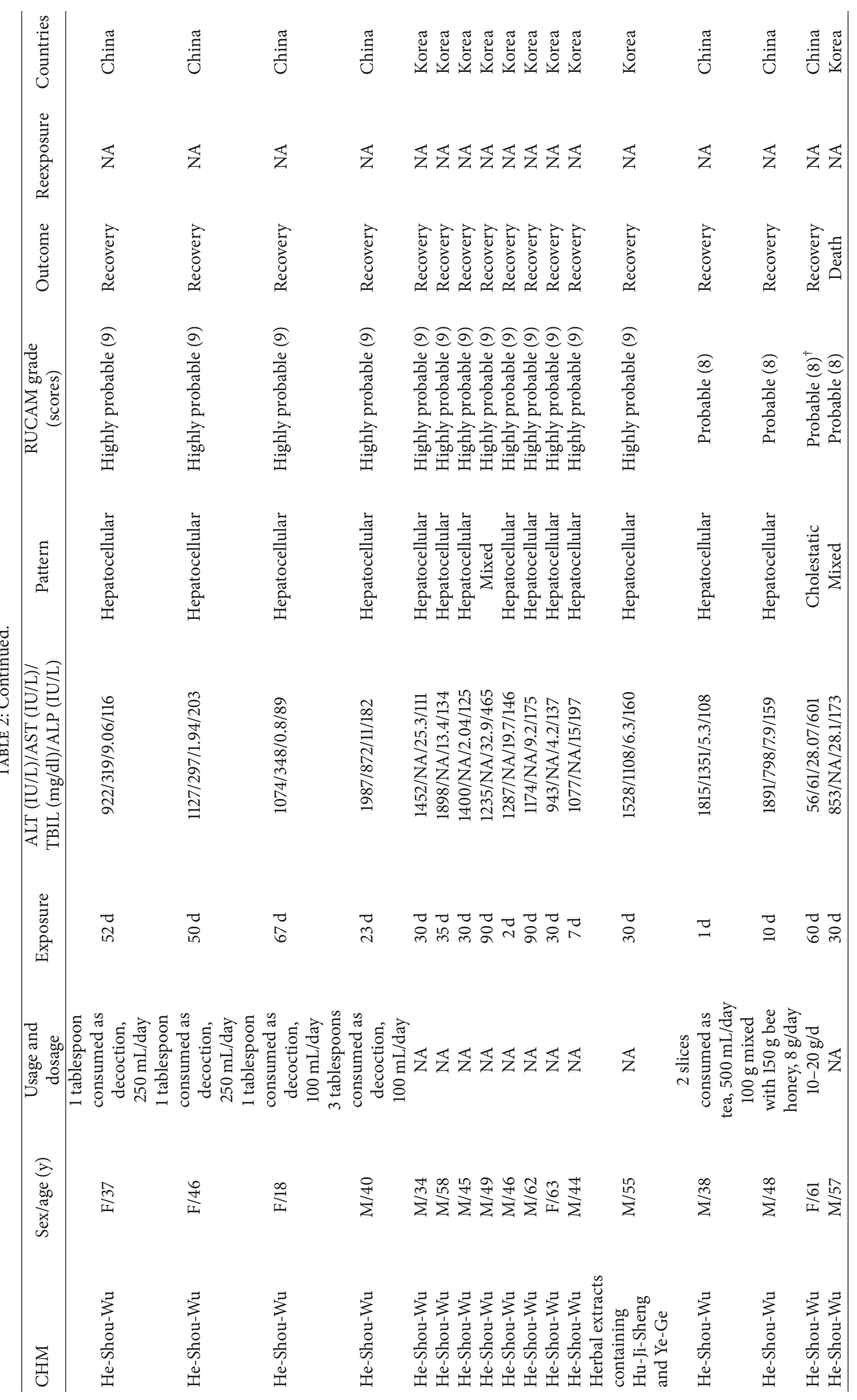




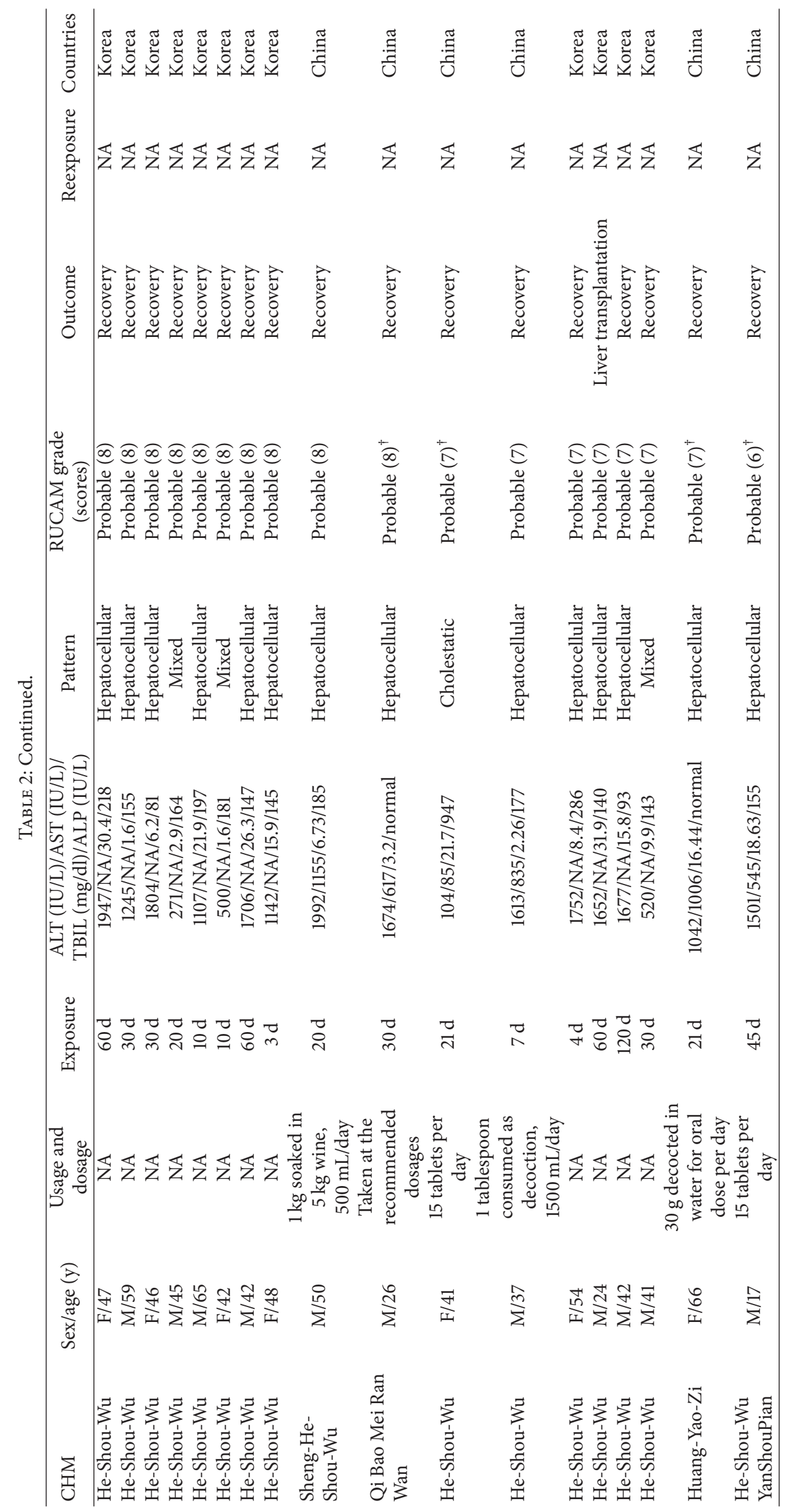




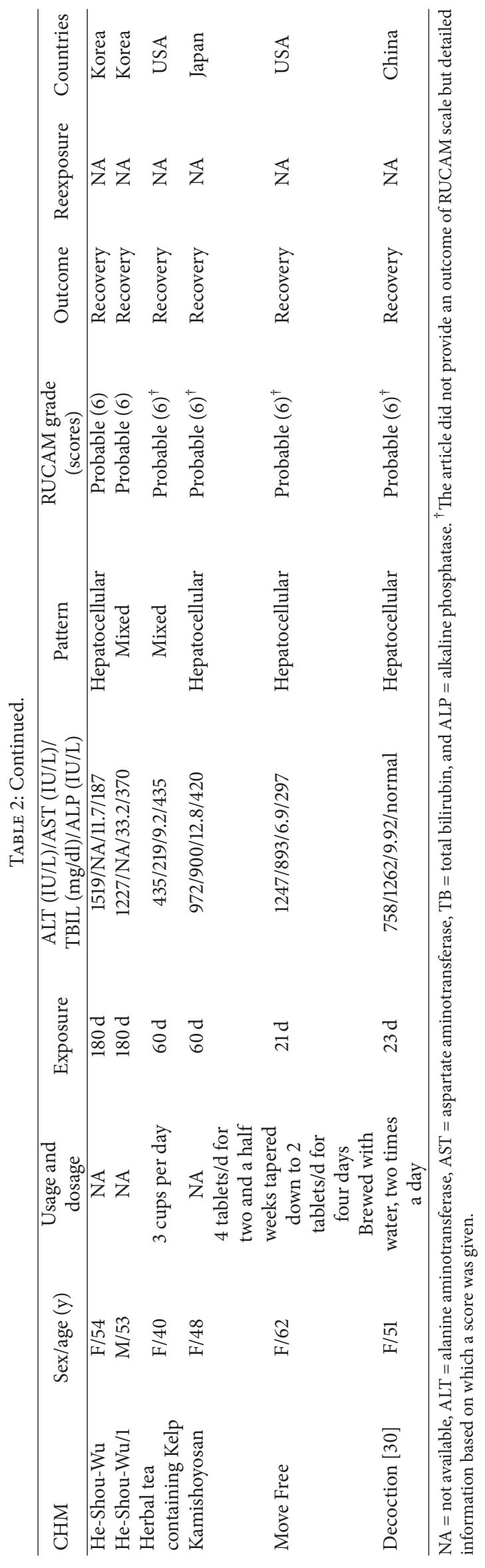


levels of highly probable or probable, indicating a high quality of causality assessment. What is more, many articles included did not provide a score of RUCAM, while the tool is widely accepted by researchers and clinicians [51-53]. The RUCAM scale is not without problems, especially for CHM induced liver injury. Previous information of $\mathrm{CHM}$ is not always available, and contamination by heavy metals and adulteration also provide challenges [54], which should be clarified before causality assessment. But it at least provides us with a framework, within which clinicians can organize the history taking and laboratory tests.

Based on our work, some suggestions were offered. First, CHM identified with reported hepatotoxicity should be used carefully. Secondly, since there is uncertain accuracy in determining the relationship between CHM and liver injury in most reports, we would like to adopt a strict definition of hepatotoxicity and the RUCAM scale, which contributes to excluding cases of other causes and offers causality evidence. Thirdly, when clinicians and researchers plan to report cases or carry out associated studies, it is advisable that details of causative herbs or patent drugs, like locality, botanical classification, brand names, detailed herbal ingredients and contents, and usage and dosage, should be provided at full length. What is more, it is necessary to build up a new website that provides up-to-date, comprehensive, and unbiased information about CHM induced liver injury and standardizes submission of associated information. Finally, since CHM is general designation of various kinds of herbs, we consider it improper to compare CHM with a single medicine.

In contrast with previous reviews, we adopted a high threshold of laboratory tests to avoid nonspecific liver injuries and included electronic data in China, which explained the difference from results of other reviews. Also, due to much more attention attracted in recent years, the increasing number of articles [7], and the development of diagnosis [55], our review focused on publications of the past 5 years. Of course, our review has its limitations. Above all, gray literature was not included, since cases unpublished were unavailable. A large number of publications searched failed to provide essential information associated with our inclusion criteria, which leads to missing data and may result in a risk of bias. Moreover, detailed information of herbs in the primary articles, like plant family, subfamily, species, subspecies, and locality, was unmentioned, which contributes to excluding adulteration and contamination. Finally, while the strict definition of hepatotoxicity helps us exclude unspecific liver injury, it may cause some toxic herbs to be missed. In fact, numbers of articles, especially articles in Chinese, were excluded for a different standard of diagnosis with ours.

In conclusion, CHM, especially He-Shou-Wu and those for dermatosis and osteoarthrosis, should be used carefully, and routine liver tests may be needed. Although cases have been increasingly reported, details about causative herbs need to be particularly illustrated. Liver injury from CHM is similar to that from conventional medicines in patent period, injury pattern, and prognosis. Further studies are needed on toxicity mechanisms and biomarkers, and more RUCAM should be used in future cases.

\section{Competing Interests}

The authors declare that they have no competing interests.

\section{Acknowledgments}

The authors' work was supported by the National Science and Technology Major Project of the 11th five-year plan in China (no. 2008ZX10005-006).

\section{References}

[1] M. L. Dossett, R. B. Davis, A. J. Lembo, and G. Y. Yeh, "Complementary and alternative medicine use by US adults with gastrointestinal conditions: results from the 2012 National Health Interview Survey," The American Journal of Gastroenterology, vol. 109, no. 11, pp. 1705-1711, 2014.

[2] T.-G. Liu, S.-Q. Xiong, Y. Yan, H. Zhu, and C. Yi, "Use of Chinese herb medicine in cancer patients: a survey in southwestern China," Evidence-Based Complementary and Alternative Medicine, vol. 2012, Article ID 769042, 5 pages, 2012.

[3] H.-K. Shin, S.-J. Jeong, D. S. Huang, B.-K. Kang, and M. S. Lee, "Usage patterns and adverse experiences in traditional Korean medicine: results of a survey in South Korea," BMC Complementary and Alternative Medicine, vol. 13, article 340, 2013.

[4] G. Shumer, S. Warber, S. Motohara et al., "Complementary and alternative medicine use by visitors to rural Japanese family medicine clinics: results from the international complementary and alternative medicine survey," BMC Complementary and Alternative Medicine, vol. 14, no. 1, article 360, 2014.

[5] Group of Hepatobiliary Disease of Digestive Disease Branch of Chinese Medical Association, "A multicenter survey on hospital inpatients with drug-induced acute liver injury in China," Zhong Hua Xiao Hua Za Zhi, vol. 27, no. 7, pp. 437-440, 2007 (Chinese).

[6] Y. Zhou, L. Yang, Z. L. Liao, X. Y. He, Y. Y. Zhou, and H. Guo, "Epidemiology of drug-induced liver injury in China: a systematic analysis of the Chinese literature including 21789 patients," European Journal of Gastroenterology and Hepatology, vol. 25, no. 7, pp. 825-829, 2013.

[7] R. Teschke, A. Wolff, C. Frenzel, J. Schulze, and A. Eickhoff, "Herbal hepatotoxicity: a tabular compilation of reported cases," Liver International, vol. 32, no. 10, pp. 1543-1556, 2012.

[8] R. Teschke, A. Wolff, C. Frenzel, and J. Schulze, "Review article: herbal hepatotoxicity - an update on traditional Chinese medicine preparations," Alimentary Pharmacology and Therapeutics, vol. 40, no. 1, pp. 32-50, 2014.

[9] R. Teschke, L. Zhang, H. Long et al., "Traditional Chinese Medicine and herbal hepatotoxicity: a tabular compilation of reported cases," Annals of Hepatology, vol. 14, no. 1, pp. 7-19, 2015.

[10] W.-J. Lee, H.-W. Kim, H.-Y. Lee, and C.-G. Son, "Systematic review on herb-induced liver injury in Korea," Food and Chemical Toxicology, vol. 84, pp. 47-54, 2015.

[11] G. Danan and R. Teschke, "RUCAM in drug and herb induced liver injury: the update," International Journal of Molecular Sciences, vol. 17, no. 1, article 14, 2016.

[12] J. P. Higgins and S. Green, Cochrane Handbook for Systematic Reviews of Interventions. Version 5.1.0, 2011, http://handbook.cochrane.org/. 
[13] D. Moher, A. Liberati, J. Tetzlaff, D. G. Altman, and The PRISMA Group, "Preferred reporting items for systematic reviews and meta-analyses: the PRISMA statement," International Journal of Surgery, vol. 8, no. 5, pp. 336-341, 2010.

[14] Pharmacopoeia Commission of the Ministry of Health, Pharmacopoeia of The People's Republic of China, 1st Div, China Chemical Industry Press, Beijing, China, 2015 (Chinese).

[15] X. Li, C. Qu, Q. He et al., "Acute hepatitis induced by a Chinese herbal product Qibao Meiran Wan: a case study," International Journal of Clinical and Experimental Medicine, vol. 8, no. 7, pp. 11624-11627, 2015.

[16] E. Cortez, C. Boulger, and A. Bernard, "Ban Tu Wan hepatotoxicity," BMJ Case Reports, vol. 2012, Article ID 006438, 4 pages, 2012.

[17] R. Dhanasekaran, V. Owens, and W. Sanchez, "Chinese skullcap in move free arthritis supplement causes drug induced liver injury and pulmonary infiltrates," Case Reports in Hepatology, vol. 2013, Article ID 965092, 4 pages, 2013.

[18] K. A. Jung, H. J. Min, S. S. Yoo et al., "Drug-induced liver injury: twenty five cases of acute hepatitis following ingestion of Polygonum multiflorum thunb," Gut and Liver, vol. 5, no. 4, pp. 493-499, 2011.

[19] H. Dong, D. Slain, J. Cheng, W. Ma, and W. Liang, "Eighteen cases of liver injury following ingestion of Polygonum multiflorum," Complementary Therapies in Medicine, vol. 22, no. 1, pp. 70-74, 2014.

[20] L. Viswanathan and A. Patel, "Hepatotoxicity associated with herbal tea containing kelp," ACG Case Reports Journal, vol. 1, no. 1, pp. 55-57, 2013.

[21] L. Yang, A. Aronsohn, J. Hart, and D. Jensen, "Herbal hepatoxicity from Chinese skullcap: a case report," World Journal of Hepatology, vol. 4, no. 7, pp. 231-233, 2012.

[22] H. J. Kim, H. Kim, J. H. Ahn, and H. J. Suk, "Liver injury induced by herbal extracts containing mistletoe and kudzu," Journal of Alternative and Complementary Medicine, vol. 21, no. 3, pp. 180185, 2015.

[23] H. Inoue, S. Yamazaki, M. Shimizu et al., "Liver injury induced by the Japanese herbal drug Kamishoyosan," Gastroenterology and Hepatology, vol. 7, no. 10, pp. 692-695, 2011.

[24] M. Wang, J. T. Qu, B. Dai, and C. L. Mei, "Fructus Xanthii poisoning: a case report and literature review," Zhong Guo Quan Ke Yi Xue, vol. 16, no. 2, pp. 218-220, 2013 (Chinese).

[25] C. Yuan and L. Chen, "Two cases of recurrent jaundice induced by oral use of He-Shou-Wu," Lin Chuang He Li Yong Yao, vol. 10, no. 7, article 78, 2014 (Chinese).

[26] A. H. Jiang and L. M. Yang, "One case of liver injury induced by Huang Yao Zi," Zhong Guo Bao Jian Ying Yang, no. 5, article 3187, 2014 (Chinese).

[27] W. L. Zhang, J. W. Ma, and J. Dong, "Case analysis of liver injury induced by Sheng-He-Shou-Wu," Kong Jun Yi Xue Za Zhi, vol. 2, no. 30, article 121, 2014 (Chinese).

[28] M. Yang, H. Y. Rao, and L. Wei, "Drug-induced liver injury: take care of herbal and dietary supplements," Jian Kang Guan Li, vol. 12, pp. 57-60, 2014 (Chinese).

[29] X. F. Ren and J. M. Xu, "A clinical study on cases with traditional chinese medicine induced liver injury," An Hui Yi Yao, vol. 19, no. 10, pp. 1997-2000, 2015 (Chinese).

[30] L. Mao, S. Q. Li, and Z. Q. Liang, "Drug-induced hepatitis due to oral traditional chinese medicine decoction," Zhong Guo Yi Yao, vol. 1, no. 8, pp. 121-122, 2013 (Chinese).
[31] G. H. Wei, "Liver injury caused by traditional chinese medicine for psoriasis," Jian Kang Bi Du ZaZhi, vol. 2, article 163, 2011 (Chinese).

[32] J. Ma, L. Zheng, Y.-S. He, and H.-J. Li, "Hepatotoxic assessment of Polygoni Multiflori Radix extract and toxicokinetic study of stilbene glucoside and anthraquinones in rats," Journal of Ethnopharmacology, vol. 162, pp. 61-68, 2015.

[33] G. P. Lv, L. Z. Meng, D. Q. Han, H. Y. Li, J. Zhao, and S. P. Li, "Effect of sample preparation on components and liver toxicity of Polygonum multiflorum," Journal of Pharmaceutical and Biomedical Analysis, vol. 109, pp. 105-111, 2015.

[34] Y. Wang, T. Han, L.-M. Xue et al., "Hepatotoxicity of kaurene glycosides from Xanthium strumarium L. fruits in mice," Pharmazie, vol. 66, no. 6, pp. 445-449, 2011.

[35] J. Wang, L. Ji, H. Liu, and Z. Wang, "Study of the hepatotoxicity induced by Dioscorea bulbifera L. rhizome in mice," BioScience Trends, vol. 4, no. 2, pp. 79-85, 2010.

[36] X.-X. Li, F.-Y. Du, H.-X. Liu, J.-B. Ji, and J. Xing, "Investigation of the active components in Tripterygium wilfordii leading to its acute hepatotoxicty and nephrotoxicity," Journal of Ethnopharmacology, vol. 162, pp. 238-243, 2015.

[37] J. Li, F. Shen, C. Guan et al., "Activation of Nrf2 protects against triptolide-induced hepatotoxicity," PLOS ONE, vol. 9, no. 7, Article ID e100685, 2014.

[38] N. P. Chalasani, P. H. Hayashi, H. L. Bonkovsky, V. J. Navarro, W. M. Lee, and R. J. Fontana, "ACG clinical guideline: the diagnosis and management of idiosyncratic drug-induced liver injury," The American Journal of Gastroenterology, vol. 109, no. 7, pp. 950-966, 2014.

[39] W. M. Lee, R. H. Squires Jr., S. L. Nyberg, E. Doo, and J. H. Hoofnagle, "Acute liver failure: summary of a workshop," Hepatology, vol. 47, no. 4, pp. 1401-1415, 2008.

[40] A. Reuben, D. G. Koch, and W. M. Lee, "Drug-induced acute liver failure: results of a U.S. multicenter, prospective study," Hepatology, vol. 52, no. 6, pp. 2065-2076, 2010.

[41] S. H. Bae, D. H. Kim, Y. S. Bae et al., "Toxic hepatitis associated with Polygoni multiflori," The Korean Journal of Hepatology, vol. 16, no. 2, pp. 182-186, 2010.

[42] P. Ou, Y. Chen, B. Li et al., "Causes, clinical features and outcomes of drug-induced liver injury in hospitalized patients in a Chinese tertiary care hospital," SpringerPlus, vol. 4, article 802, 2015.

[43] G. P. Lv, L. Z. Meng, D. Q. Han, H. Y. Li, J. Zhao, and S. P. Li, "Effect of sample preparation on components and liver toxicity of Polygonum multiflorum," Journal of Pharmaceutical and Biomedical Analysis, vol. 109, pp. 105-111, 2015.

[44] W. I. Cheung, M. L. Tse, T. Ngan et al., "Liver injury associated with the use of Fructus Psoraleae (Bol-gol-zhee or Bu-gu-zhi) and its related proprietary medicine," Clinical Toxicology, vol. 47, no. 7, pp. 683-685, 2009.

[45] K. Wang, Z. Song, H. Wang, Q. Li, Z. Cui, and Y. Zhang, "Angelica sinensis polysaccharide attenuates concanavalin A-induced liver injury in mice," International Immunopharmacology, vol. 31, pp. 140-148, 2016.

[46] Y.-M. Liu, L.-L. Zhu, R. Li et al., "Xijiao Dihuang Decoction (fbft) and Rehmannia glutinosa Libosch. protect mice against lipopolysaccharide and tumor necrosis factor alpha-induced acute liver failure," Chinese Journal of Integrative Medicine, 2015.

[47] S. Wu, Y. Yue, H. Tian et al., "Carthamus red from Carthamus tinctorius L. exerts antioxidant and hepatoprotective effect against $\mathrm{CCl}_{4}$-induced liver damage in rats via the $\mathrm{Nrf2}$ pathway," Journal of Ethnopharmacology, vol. 148, no. 2, pp. 570-578, 2013. 
[48] B. Zhang, Q. Zhang, M. Liu et al., "Increased involvement of Panax notoginseng in the mechanism of decreased hepatotoxicity induced by Tripterygium wilfordii in rats," Journal of Ethnopharmacology, vol. 185, pp. 243-254, 2016.

[49] Livertox, "Chinese and Other Asian Herbal Medicines," 2016, http://livertox.nih.gov/.

[50] Hepatox, http://www.hepatox.org/chPatentDrug.

[51] J. Rochon, P. Protiva, L. B. Seeff et al., "Reliability of the Roussel Uclaf Causality Assessment Method for assessing causality in drug-induced liver injury," Hepatology, vol. 48, no. 4, pp. 11751183, 2008.

[52] R. Teschke, C. Frenzel, J. Schulze, and A. Eickhoff, "Herbal hepatotoxicity: challenges and pitfalls of causality assessment methods," World Journal of Gastroenterology, vol. 19, no. 19, pp. 2864-2882, 2013.

[53] R. Teschke, A. Wolff, C. Frenzel, A. Schwarzenboeck, J. Schulze, and A. Eickhoff, "Drug and herb induced liver injury: council for International Organizations of Medical Sciences scale for causality assessment," World Journal of Hepatology, vol. 6, no. 1, pp. 17-32, 2014.

[54] R. Teschke, A. Eickhoff, and J. Schulze, "Drug- and herbinduced liver injury in clinical and translational hepatology: causality assessment methods, quo vadis?" Journal of Clinical and Translational Hepatology, vol. 1, no. 1, pp. 59-74, 2013.

[55] T. Tran and W. M. Lee, "DILI: new insights into diagnosis and management," Current Hepatitis Reports, vol. 12, no. 1, pp. 53-58, 2013. 


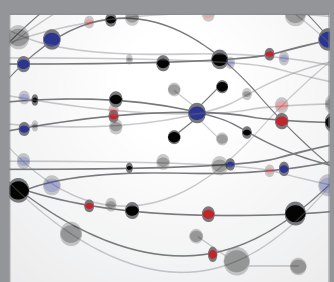

The Scientific World Journal
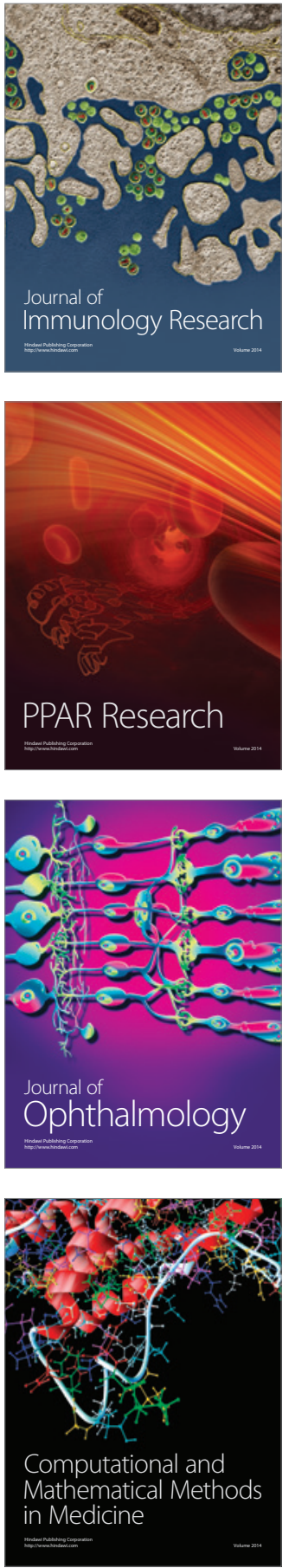

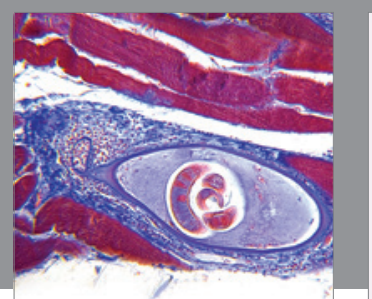

Gastroenterology Research and Practice

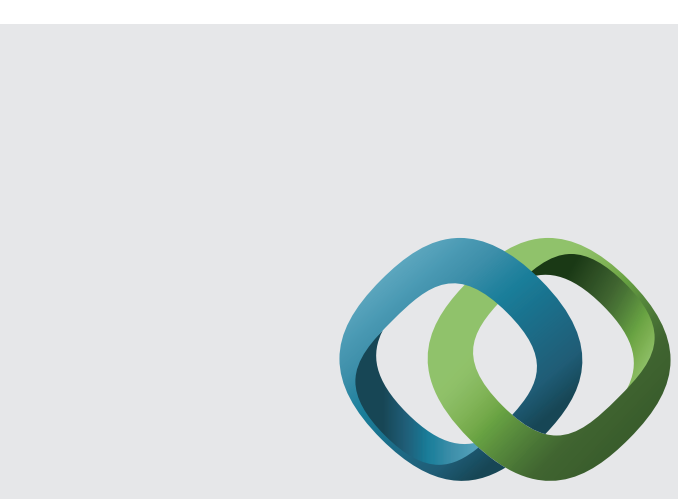

\section{Hindawi}

Submit your manuscripts at

http://www.hindawi.com
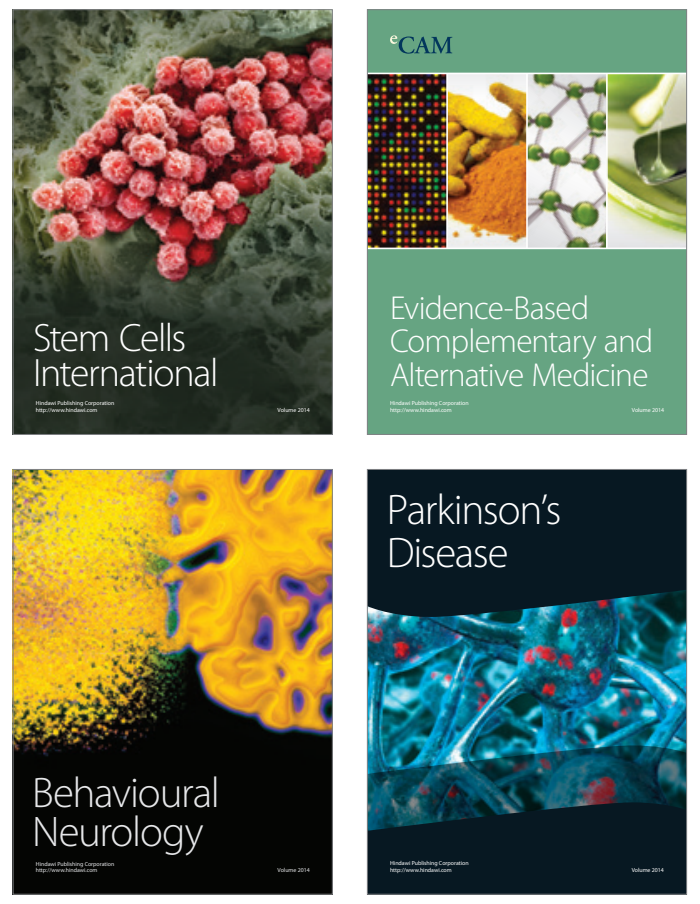
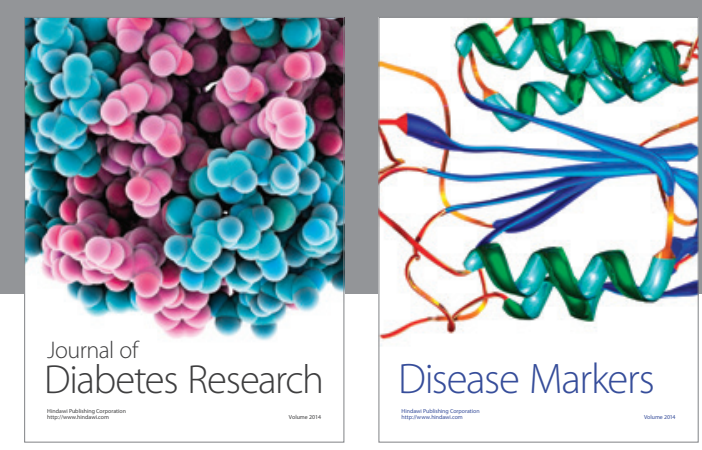

Disease Markers
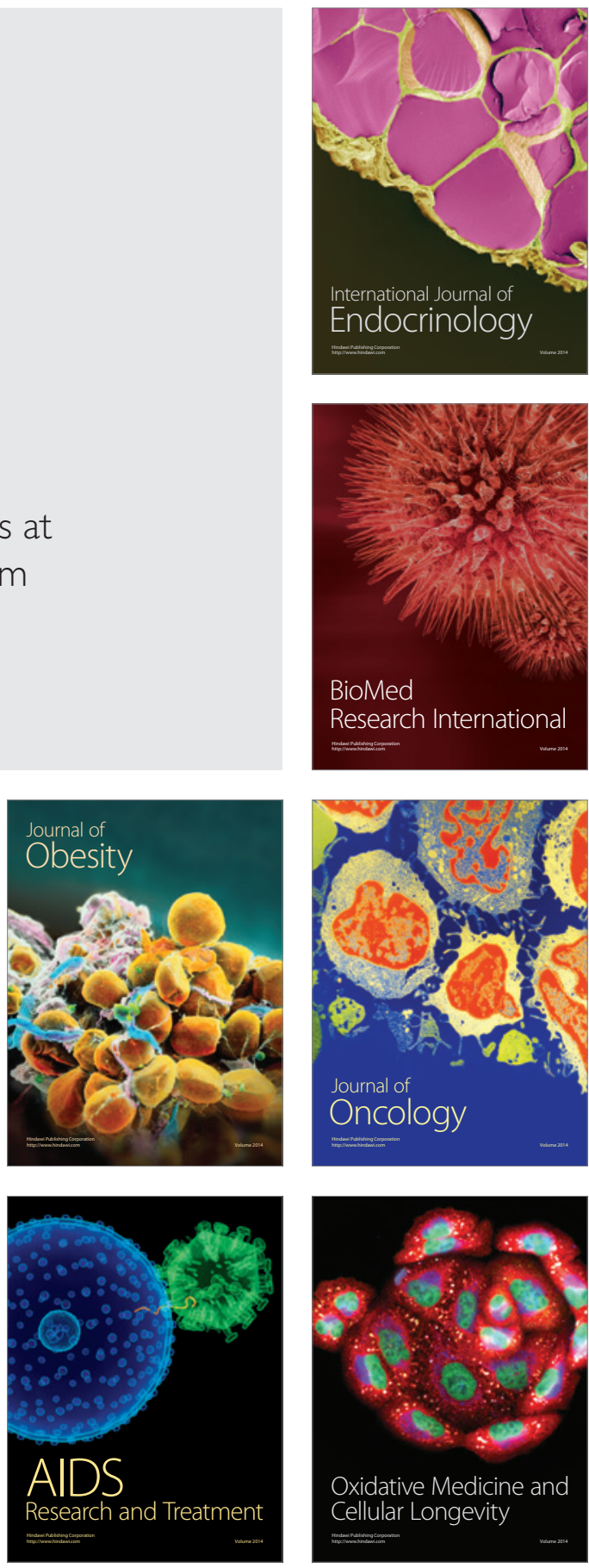